\title{
Reduction of speckling noise of SAR Images using Dual Tree Complex Wavelet (DTCW) and Shearlet Transforms
}

\author{
J. Deny ${ }^{1}$, M. Sundarajan ${ }^{2}$, R. Raja Sudharsan ${ }^{3}$, E. Muthukumaran ${ }^{4}$, B. Perumal ${ }^{5}$ \\ \{j.deny@klu.ac.in ${ }^{1}$, msrajan69@gmail.com $^{2}$,rajasudharsan@klu.ac.in ${ }^{3}$, reachmek@gmail.com ${ }^{4}$, \\ perumal@klu.ac.in $\left.{ }^{5}\right\}$ \\ Kalasalingam Academy of Research and Education, Krishnan koil, Virudhunagar, India ${ }^{1,3, \& 5}$ \\ Bharath Institute of Higher Education and Research, Chennai, India ${ }^{2}$ \\ Dr. B. R Ambedkar Institute of Technology, Port Blair, Andaman \& Nicobar Islands ${ }^{4}$
}

\begin{abstract}
The Synthetic Aperture Radar (SAR) images are difficult to interpret owing to the multiplicative speckle noise from coherent acquisition systems. Therefore, despeckling of SAR images always plays a primary pre-processing task in SAR image processing. There are many methods using various spatial domain filters and transform domain algorithms which focus on speckle reduction but not all methods can preserve the image edge features. The article suggests a de-speckling algorithm via sparse representation using combined Shearlet Transform and DTCW transform which possess direction selectivity and shift invariant property. The experimental results, the suggested method has better PSNR, ENL, and EPI values than the existing state of art methods. The proposed methodology not only preserves the edges, also improves visual effect by enhancing the texture of SAR images.
\end{abstract}

Keywords: Synthetic Aperture Radar (SAR), Speckle Noise, Shearlet Transform, DTCW Transform, Sparse Coefficients.

\section{Introduction}

The SAR microwave imagery has become one of the most beneficial applications over the optical satellite symbolism on account of its capacity to work in the entire climate conditions. It has different applications, for example, namely: agriculture, remote sensing, land applications, potential applications, flood mapping, soil moisture, forestry, terrain analysis and so on [1]. SAR images obtained from Synthetic Aperture Radar are very difficult to understand and analyse because of presence of multiplicative noise called speckle noise [2]. The resolution of images is affected by the nearness of specked images. These specked images are difficult for human interpretation and hence there is a need to remove noise from SAR images. De-speckling is the pre-processing step of Automatic Target Recognition followed by detection, classification and recognition [3].

To overcome the above-mentioned drawbacks of spatial domain methods, transform domain methods were introduced. Wavelet transform is used for de-speckling of SAR images by several researchers. There is comprehensive examination of wavelet based de-speckling approaches. A wavelet transform method have better time frequency characteristics than the Fourier transform analysis and also preserves texture features and edges efficiently. The 
wavelet transform based methods cannot sparsely represent high dimensional functions [4]. To overcome the above limitation, scholars proposed many multiscale geometric transformations such as Contour let, Ridge let and Curve let Transforms. But these transforms for SAR image de-speckling blur the image edges and produce artificial images. In order to overcome the above drawbacks researchers introduced Shear let Transform which has flexible direction selectivity property [5].

\section{1) Modelling of Speckle Noise}

Though multiplicative clamour which is brought about by de-staged echoes from a backscattered signal is mind-boggling to demonstrate and furthermore hard to lessen . Dot is actually not a clamour but rather commotion like a granular example. The multiplicative spot clamour is as

$$
Z(k, l)=S(k, l) N(k, l)
$$

Where, $Z(k, l)$ denotes the intensity format of corrupted SAR image, $S(K, l)$ denotes the noise free SAR image that has to be recovered and $N(k, l)$ denotes the multiplicative speckle component. The multiplicative clamour is thusly changed over into added substance commotion which is given by

$$
Z(k, l)=S(k, l)+N(k, l)
$$

The log change yields undesired impacts in the SAR pictures since the mean of the logchanged dot isn't zero. The proposed methodology comprises of combining Shearlet transform with Dual Tree Complex Wavelet Transform which possess both direction selectivity and shift invariance for efficient speckle reduction and extracting the sparseness of the transform coefficients to convert the denoising process into an optimization model.

\section{Proposed Methodology}

The proposed technique has been clarified in beneath algorithm. Proposed STDTCWT algorithm for noise reduction given better results than existing algorithms.

\section{a. Shearlet Transform}

The execution of Shearlet transform is simple and flexible than multi resolution analysis transforms like Curvelet, Contourlet and Ridgelet transforms. For a 2-D signal, the Shearlet functions are,

$$
A_{A B(\varphi)}=\left\{\varphi_{i, j, k(x)}=|\operatorname{det} A|^{\frac{j}{2}} \varphi\left(B^{l} A^{j} x-k\right)\right.
$$




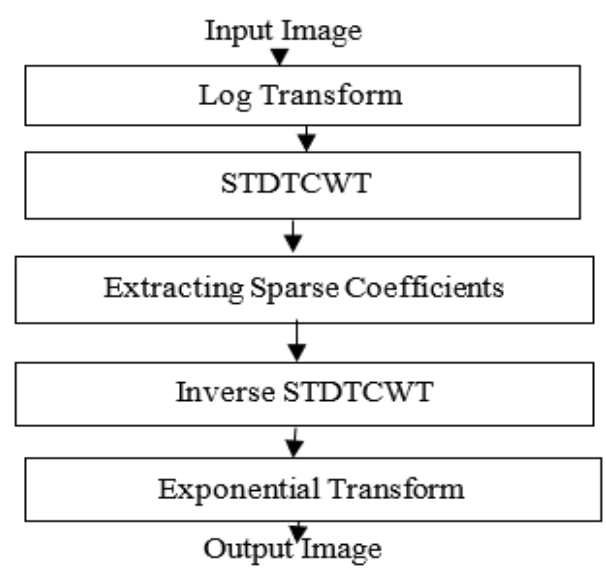

Fig. 1.Pipeline of the proposed methodology where $\psi \in L^{2}\left(R^{2}\right), A, B$ is 2-D matrices and $|\operatorname{det} B|=1$.

Let $\mathbf{A}=\mathbf{A} 0=\left(\begin{array}{ll}4 & 0 \\ 0 & 2\end{array}\right)$ be the anisotropic dilation matrix $\mathbf{B}=\mathbf{B} 0=\left(\begin{array}{ll}1 & 1 \\ 0 & 1\end{array}\right)$ be the shear matrix. Thewavelet functions,

$$
\begin{aligned}
& \left(\begin{array}{c}
\varphi_{j, i k}^{[0]}(x)=2^{8 j \backslash 2} \varphi^{[0]}\left(B_{0}^{j} A_{0}^{j} x-k\right) \\
j j \geq 0,-2^{j} \leq l \leq 2^{j}-1, k \in Z^{2}
\end{array}\right) \\
& S H \psi=\left\langle f_{g} \mid \varphi_{j_{2} l_{2} k}^{(d)}\right\rangle
\end{aligned}
$$

Where, $j \geq 0, l=-2 j \sim 2 j-1, \boldsymbol{k} \in Z^{2}, d=0,1$.

The proposed method uses the move invariant Shearlet change to accomplish SAR picture denoising dependent on meagre portrayal.

\section{b. DTCW Transform}

The DTCWTransform of a two-Dimensional image $(k, l)$ gives anestimation of sub-band and 6-directional particular sub-groups at all level, are firmly arranged at edges $\pm 15^{\circ}, \pm 45^{\circ}$ and $\pm 75^{\circ}$. Accordingly, the denoising must be done distinctly in the 6-directional sub-groups. After applying DTCWTransform on equ. (2), we obtain,

$$
d_{j}^{i}(k, l)=x_{j}^{i}(k, l)+n_{j}^{i}(k, l)(6)
$$

Where, $d_{j}^{i}(k, l), x_{j}^{i}(k, l)$ and $n_{j}^{i}(k, l)$ is the $(k, l)^{t h}$ DTCW Transform coefficient of the logchanged watched power, the first force, and the comparing commotion segment, individually, at level $j$ with direction $i$. Also, the DTCW Transform wavelet coefficients are modelled using a bivariate Cauchy PDF. For N-dimensional vector,

$$
(\mathrm{x})=\frac{\gamma \mathrm{r}\left[\frac{1+\mathbb{N}}{2}\right]}{\left.\left[\pi(\mid x]^{2}+\gamma^{2}\right)\right]^{(1+N) / 2}}
$$

gamma function $\Gamma(\mathrm{L})=\int_{0}^{\infty} t^{L-1} e^{-1} \mathrm{dt}$. 


\section{Results and Discussion}

The input image taken for carrying experimental results is MSTAR dataset. The performance of suggested STDTCWT technique is compared separately with the Shearlet and DTCWT transforms

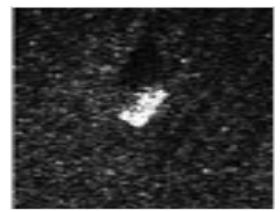

ca)

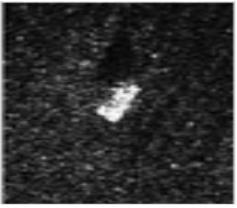

(b)

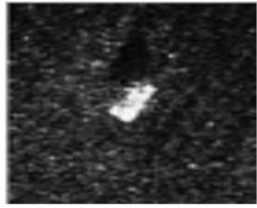

(c)

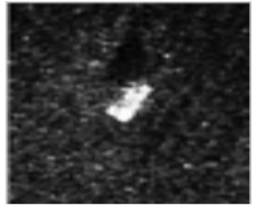

(a)

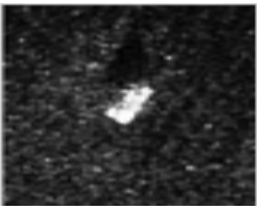

ie)

Fig.2 (a) Input Image (b) Input Image with noise (c) De-speckled Image using Shearlet Transform (d) De-speckled Image using DTCWT (e) De-speckled Image using proposed STDTCWT

Table1.Performance comparison table for BRDM.

\begin{tabular}{|l|l|l|l|l|}
\hline Input Image & Parameters & Shearlet & DTCWT & STDTCWT \\
\hline \multirow{3}{*}{} & PSNR & 42.6839 & 32.5079 & 44.1264 \\
\cline { 2 - 5 } & ENL & 24.1030 & 21.8284 & 24.1284 \\
\cline { 2 - 5 } & EPI & 0.9872 & 0.9321 & 0.9875 \\
\cline { 2 - 5 } & SSI & 0.7972 & 0.8701 & 0.7967 \\
\cline { 2 - 5 } & SD & 30.7696 & 31.2182 & 30.7623 \\
\hline
\end{tabular}

Table 2. Performance comparison table for various images of MSTAR Dataset

\begin{tabular}{|l|l|l|l|l|l|l|}
\hline Input Image & Methods & PSNR & ENL & EPI & SSI & SD \\
& & & & & & \\
\hline & ShearletDTCWT & 36.3456 & 22.7865 & 0.9453 & 0.7642 & 30.2343 \\
& Proposed & 37.2987 & 23.8764 & 0.9234 & 0.7245 & 30.4321 \\
& & 39.5643 & 24.1298 & 0.9934 & 0.7124 & 30.1536 \\
\hline & ShearletDTCWT & 35.5674 & 22.5467 & 0.9453 & 0.7678 & 30.4567 \\
& Proposed & 36.8976 & 23.3445 & 0.9789 & 0.7896 & 30.6789 \\
& & 39.3455 & 25.6785 & 0.9997 & 0.7299 & 30.2435 \\
\hline & ShearletDTCWT & 36.7438 & 23.7864 & 0.9123 & 0.6934 & 29.9872 \\
& Proposed & 39.2345 & 24.6512 & 0.9123 & 0.6776 & 28.2345 \\
& & 40.2376 & 26.9034 & 0.9278 & 0.6100 & 28.1000 \\
\hline & & & & & \\
\hline & ShearletDTCWT & 41.5420 & 23.7230 & 0.8346 & 0.7256 & 29.4663 \\
& Proposed & 42.2563 & 25.9987 & 0.8593 & 0.7377 & 28.3678 \\
& & 43.9892 & 26.8719 & 0.8721 & 0.7177 & 27.2356 \\
\hline
\end{tabular}




\begin{tabular}{|l|l|l|l|l|l|l|}
\hline$*$ & ShearletDTCWT & 42.6839 & 24.1030 & 0.9872 & 0.7972 & 30.7996 \\
& Proposed & 42.5079 & 21.1284 & 0.9321 & 0.8701 & 31.2182 \\
& & 44.1264 & 24.1284 & 0.9875 & 0.7967 & 30.7623 \\
\hline
\end{tabular}

The performance comparison table shows that the proposed methodology gives higher PSNR, ENL and EPI values and lower SSI and SD values.

\section{Conclusion}

This work proposed a SAR Image De-speckling using combined Shearlet and DTCW transforms provide both shift invariant and directional selectivity properties. The performance of de-speckling algorithm is compared with Shearlet and DTCWT transforms. The parameters PSNR, ENL and EPI values of the proposed method are high and SSI and SD values are lesser and it is compared to existing de-speckling algorithms, results in stronger speckle-noise reduction and greater visual quality.

\section{References}

[1] R.Touzi, "A Review of Speckle Filtering in the Context of Estimation Theory, IEEE Trans. Of Geoscience and Remote Sensing, 2002.

[2] Alin Achim, Panagiotis, Tsakalides and Anastasios Berrionos, "SAR Image Denoising via Bayesian Wavelet Shrinkage based on Heavy Tailed Modelling, IEEE Trans. Geoscience and Remote Sensing,2003

[3] Perumal Vasuki,S,Mohamed Mansoor Roomi, "Particle Swarm Optimization based despeckling and decluttering of Wavelet Packet Transformed Synthetic Aperture Radar Images" Journal of Applied Remote Sensing, 2013

[4] P.Vasuki, S.Mohamed Mansoor Roomi Automatic Noise Identification in Images Using Moments and Neural Network, "International IEEE Conference on Machine Vision and Image Processing", PSGTECH, Coimbatore, 2012.

[5] J.-S. Lee, "Digital image enhancement and noise filtering by use of local statistics," IEEE Trans. Patt. Anal. Mach. Intell., no. 2, pp.165-168, 1980.

[6] G.Maragatham,S Md Mansoor Roomi, P Vasuki, "Noise Detection in Images using Moments," Research Journal of Applied Sciences, Engineering and Technology, P307 - 314,2015.

[7] T. Bianchi, F. Argenti, and L. Alparone, "Segmentation- Based MAO despeckling of SAR images in the undecimated wavelet domain,IEEE Trans. Geosci.Remote Sensing,vol. 46.no.9.pp.2728-2742,2008

[8] P.Vasuki, S.Mohamed Mansoor Roomi, "Noise Variance Estimation in SAR Images Using Wavelets and Filter Selection " International IEEE Conference on Computing Communication and Networking Technologies, ICCCNT'2013, Vivekananda College of Engineering for Women, Tiruchengode, 2013.

[9] S. Ghael, A. Sayeed, and R. Baraniuk, "Improved wavelet denoising via empirical wiener filtering," in Proc. SPIE, vol. 5, Jul. 1997, pp. 389-399.

[10] Hua Xie ; L.E. Pierce ; F.T. Ulaby, "Despeckling SAR images using a low-complexity wavelet denoising process IEEE International Geoscience and Remote Sensing Symposium 2011

[11] Venkata Rukmini,2008, Thesis on Filter selection for Speckle noise Reduction,Thapar university,Patiala, Punjab. 
[12] Jennifier Ranjani and Thiruvengadam, 2011, Generalized SAR Despeckling based on DTCWTexploiting Interscale

[13] and Intrascale dependences, J. IEEE trans.on Geoscience and Remote sensing letters, 8,pp.551555.

[14] V. Katkovnik, A. Foi, K. Egiazarian, and J. Astola, "From local kernel to nonlocal multiplemodel image denoising," International Journal ofComputer Vision, vol. 86, no. 1, pp. 1-30, Jan. 2010

[15] A. Levin and B. Nadler. Natural image denoising: Optimally and inherent bounds. In Computer Vision and Pattern Recognition (CVPR),2011, IEEE Conference on pages 28332840.

[16] J Deny, A Sivanesh Kumar, M Sundarajan, V Khanna "Defensive against collaborative attacks by malicious nodes in MANETs: A cooperative bait detection approach", 2017 International Conference on Algorithms, Methodology, Models and Applications in Emerging Technologies (ICAMMAET) pp.1-5

[17] J Deny, A Sivanesh Kumar, N Ragupathi Muthu, B Perumal "Hybrid routing algorithm for wireless sensor networks by using improved genetic algorithm" 2017 IEEE International Conference on Intelligent Techniques in Control, Optimization and Signal Processing (INCOS'17). Pp.1-8.

[18] Xin Wan, Kezhong Zhang, S.Ramkumar,J.Deny "A Review On Electroencephalogram Based Brain Computer Interface For Elderly Disabled" IEEE Access Journal , PP. 36380-36387, Volume 7, Issue 1.

[19] J.Deny ,N.Sivasankari, "Biometric Security in Military Application ” Journal of Engineering Procedia ELSEVIER,Vol.38,pp-1138-1144, July 2012,ISSN No: 1877-7058.

[20] D.Manojkumar, K SenthilKumar, R Veera Amudhan, G Vasantha Moorthi, Dr.J.Deny, "Improve Security in IOT Using Blowfish Algorithm" International Journal of Pure and Applied Mathematics Volume 119 No. 12 2018, pp.16261-16267.

[21] Dr.J.Deny, E.Muthukumaran, S.Ramkumar, S.Kartheesawaran "Extraction of Respiratory Signals and Motion Artifacts from PPG Signal Using Modified Multi Scale Principal Component Analysis" International Journal of Pure and Applied Mathematics Volume 119 No. 12 2018, pp. 13719-13727.

[22] Dr.E.Muthukumaran. Dr.J.Deny, Dr.B.Perumal,G. Suseendran., Dr. D.Akila “A narrative NonInvasive Diagnostic loom Based by the side of correlation of Nasal set Rhythm in addition to customary Three Radial Pulses Measurement" IOP Conf. Series: Journal of Physics: Conf. Series 1228 (2019) 012075.

[23] S. Darwin, T.S. Arun Samuel, E. Muthu Kumaran, J. Deny, "Mathematical Modeling and Simulation of Junction less Dual Material Double Gate MOSFET with Various Work Functions", International Journal of Innovative Technology and Exploring Engineering (IJITEE) ISSN: 2278-3075, Volume-8 Issue-12, October 2019, pp. 3457- 3460.

[24] Sudharsan, RR, J. Deny, E. Muthu Kumaran, and A. Sharon Geege. "An Analysis of Different Biopotential Electrodes Used for Electromyography", 2020.

[25] Deny, J., and Sudharsan, RR. "Block Rearrangements and TSVs for a Standard Cell 3D IC Placement." In Intelligent Computing and Innovation on Data Science, pp. 207-214. Springer, Singapore, 2020.

[26] Sudharsan, RR, and J. Deny. "Field Programmable Gate Array (FPGA)-Based Fast and LowPass Finite Impulse Response (FIR) Filter" In Intelligent Computing and Innovation on Data Science, pp. 199-206. Springer, Singapore, 2020.

[27] Sudharsan, R.R., Deny, J., Muthukumaran, E. et al. FPGA based peripheral myopathy monitoring using MFCV at dynamic contractions. J Ambient Intell Human Comput (2020). https://doi.org/10.1007/s12652-020-02363-7

[28] J.Deny, Dr.M.Sundhararajan"Survey of Texture Analysis Using Histogram in Image Processing” International Journal of Applied Engineering Research (IJAER) Vol 26, pp .87378739. 
[29] J.Deny, Dr.M.Sundhararajan "Review of Color Image Segmentation Using K-means and Super-Pixel Algorithm" International Journal of Digital Communication and Networks, Vol. 2, Issue 12, pp: 4-8.

[30] Deny John Samuvel, B. Perumal, Muthukumaran Elangovan, "Music recommendation system based on facial emotion recognition, Publicado en 3C Tecnología. Special Issue - March 2020 . 